\title{
The Peristaltic Transport of MHD Powell - Eyring Fluid through Porous Medium in a Symmetric Channel with Slip Condition
}

\author{
Hayat Ali ${ }^{1}$, Ahmed M. Abdulhadi ${ }^{2}$ \\ ${ }^{1}$ University of Bagdad, Department of Mathematic, Baghdad, Iraq \\ ${ }^{2}$ University of Bagdad, Department of Mathematic, Baghdad, Iraq
}

\begin{abstract}
This paper is devoted to investigate the peristaltic flow of MHD Powell- Eyring model through porous medium during two dimensional symmetric channels. Slip conditions on the walls are taken into consideration. The basic governing equations; mass and momentum equations modeled with respect to wave frame. Solution technique to find the closed form solution for the resulted dimensionless differential equation is hold depending on long wavelength approximation and low Renold assumption. The influences of various involved parameters are illustrated via graphs in details. All the mathematical results are obtained by applying Mathematica 9 program.
\end{abstract}

Keywords: Peristaltic Transport, Powell- Eyring Fluid, MHD Fluid, Porous Medium

\section{Introduction}

The phenomenon in which the fluid transport as a contraction or expansion progressive waves along the length of a distensible tube is called peristalses. It can be recognized in physiological processes like chime movement in intestinal tract, blood flow in lymphatic, urine passage from kidney to the bladder, blood flow in small vessels. Also it is a key role in biomedical engineering, chemical processes, medical industry and locomotion of worm. The essential studies in this topic are done by Latham [1], and Shapiro et al.[2] through theoretical and experimental approaches. An important observation can be made from the aforementioned application that is the fluid used in these applications is non Newtonian fluids. Moreover non- Newtonian fluids as polymer melts, blood, fruit juices, ceramics, multigrade oil, and a lot others [3] can be characterize according to its own "constitutive equation" the relationship between the shear stress and the rate of deformation. Eyring -Powell model is considered as an advantageous model than the other nonNewtonian fluid model since it is deduced from kinetic theory of liquids not the empirical relation, also it correctly reduces to Newtonian behavior for low and high shear rates. For this purpose many researchers adopted the connection of peristaltic transport and Non-Newtonian fluid as a title for their works (see [4], [5], [6]). A magnetic field when applied to a moving conducting fluid it will induce electromagnetic field which produces a force named by Lorentz force opposite the fluid motion. In connection with peristaltic flow is based of applications and process like blood pumping machines, cancer therapy, biomedical engineering, industry, power generators, electrostatic precipitation, magnetic resonance imaging (MRI) for brain diagnosis, geophysics and many others. Attempted related the peristaltic flow under the effect of magnetic fluid in different situations consulted by the studies [4], [6]-[9]. On the other hand transport through porous medium is also helpful and applicable in different aspect such as geomechanics, biomechanics, industry filtration of fluids, seepage of water in river beds, functioning of human lung and others. These applications inspired numerous researchers to investigate the combine effect of porous medium and magnetic field on the fluid flows in various geometries and situations.(see [10],[11]). Slip conditions has a vital role in many applications rather than the no- slip condition like modern material industry (polymer industry) which given as a microscopic wall slip, engineering, medical application (polishing artificial heart), and technological process. Aforementioned application reported through studies discussed the influences of slip conditions on peristaltic flow of non- Newtonian fluids. Hina. [12] discussed the effect of heat and mass transfer on the peristaltic flow for MHD Eyring- Powell fluid taken into consideration slip condition. Hayat et al.[13] analyzed the effect of slip condition on peristaltic flow of Eyring- Powell fluid. Veerakrishna. et al. [9] detected the heat transfer on peristaltic flow of Williamson fluid model in an inclined symmetric channel with slip condition. Abbas et al.[7] investigate the simultaneous effect of slip and MHD on peristaltic blood flow of Jeffery fluid model in a nonuniform porous channel. Tanveer et al.[8] studies the influence of an inclined magnetic field on peristaltic transport of hyperbolic tangent nanofluid in inclined channel having flexible walls. The aim of this study is to investigate the combination effects of magnetic field and porous medium on peristaltic transient of Non-Newtonian fluid in a symmetric channel with slip condition depending on shear stress. The non- Newtonian fluid is obeying Powell- Eyring fluid model. The investigation is done by implementing the assumption of long wavelength and low Renold number. The flow's governing equation formulated by employing the wave frame. Closed expression for stream function, velocity axial and pressure gradient have been determined also numerical integration of pressure rise per unit wave obtained with help of series approximation. Finally graphical analysis has been done to figure out the effect of some interesting parameters involving in the above functions in details. 


\section{International Journal of Science and Research (IJSR) \\ ISSN (Online): 2319-7064}

Index Copernicus Value (2016): 79.57 | Impact Factor (2015): 6.391

\section{Mathematical Formulation of the Problem}

Consider the peristaltic transport of an incompressible viscous fluid flows through a porous medium in a symmetric horizontal channel of width $2 d$. The fluid is under the effect of magnetic field of constant strength $\beta_{0}$ applied in transverse direction to the flow. The magnetic Renold number is taken small and thus the induced magnetic field is neglected. The flow is induced by a sinusoidal peristaltic wave along the channel flexible walls with wave speed $c$. The Cartesian coordinates are considered in such manner that the $\bar{X}$ - axis was taken along the central line of the channel while the $\bar{Y}$ - axis is normal to it. Let $\bar{U}(\bar{X}, \bar{Y}, \bar{t})$ and $\bar{V}(\bar{X}, \bar{Y}, \bar{t})$ are represent the components of velocity in the axial $\bar{X}$ and $\bar{Y}$ directions respectively. The structure of wall geometry is described as

$$
\bar{I}= \pm \bar{H}(\bar{X}, \bar{f})= \pm\left(d+b \sin \frac{2 \pi}{\lambda}(\bar{X}-c \bar{f})\right)
$$

Here $\bar{t}$ is the time, $b$ the wave amplitude, $d$ the mean half width of the channel, $\lambda$ is the wavelength, $c$ the velocity of the peristaltic wave, $\pm \bar{H}(\bar{X}, \bar{t})$ the displacement of upper and lower walls respectively.

The fluid is obeying the Powell- Eyring model and the Cauchy stress tensor $\bar{\tau}$ of it is given as follows [13]

$$
\begin{aligned}
& \bar{\tau}=-\bar{P} I+\bar{S} \\
& \bar{S}=\left[\mu+\frac{1}{\beta \dot{\gamma}} \sinh ^{-1}\left(\frac{\dot{\gamma}}{c_{1}}\right)\right] A_{1} \\
& \dot{\gamma}=\sqrt{\frac{1}{2} \operatorname{trac}\left(A_{1}\right)^{2}} \\
& A_{1}=\nabla \vec{V}+(\nabla \vec{V})^{T}
\end{aligned}
$$

Where $\bar{S}$ express the extra stress tensor, $I$ the identity tensor, $\vec{\nabla}=(\partial \bar{X}, \partial \bar{Y}, 0)$ the gradient vector, $\left(\beta, c_{1}\right)$ the material parameters of Powell-Eyring fluid, $\bar{P}$ the pressure of the fluid, and $\mu$ the dynamic viscosity. The term $\sinh ^{-1}$ is approximated as

$\sinh ^{-1}\left(\frac{\dot{\gamma}}{c_{1}}\right)=\frac{\dot{\gamma}}{c_{1}}-\frac{\dot{\gamma}^{3}}{6 c_{1}{ }^{3}},\left|\frac{\dot{\gamma}^{5}}{c_{1}{ }^{5}}\right|<<1$

The formulation of governing equations (i.e. the continuity and the motion equations) with respect to laboratory frame $(\bar{X}, \bar{Y}, \bar{t})$ are given by

$$
\frac{\partial \bar{U}}{\partial \bar{X}}+\frac{\partial \bar{V}}{\partial \bar{Y}}=0
$$

The $\bar{X}$ - component of momentum equation

$$
\begin{gathered}
\rho\left(\frac{\partial \bar{U}}{\partial \bar{t}}+\bar{U} \frac{\partial \bar{U}}{\partial \bar{X}}+\bar{V} \frac{\partial \bar{U}}{\partial \bar{Y}}\right)=-\frac{\partial \bar{P}}{\partial \bar{X}}+\frac{\partial \bar{S}_{\overline{X X}}}{\partial \bar{X}}+\frac{\partial \bar{S}_{\overline{X Y}}}{\partial \bar{Y}} \\
-\sigma \beta_{0}^{2} \bar{U}-\frac{\mu}{\kappa_{0}} \bar{U}
\end{gathered}
$$

$$
\begin{aligned}
\rho\left(\frac{\partial \bar{V}}{\partial \bar{t}}+\bar{U} \frac{\partial \bar{V}}{\partial \bar{X}}\right. & \left.+\bar{V} \frac{\partial \bar{V}}{\partial \bar{Y}}\right)=\frac{\partial \bar{P}}{\partial \bar{Y}}+\frac{\partial \overline{S_{\overline{Y X}}}}{\partial \bar{X}}+\frac{\partial \bar{S}_{\overline{Y Y}}}{\partial \bar{Y}} \\
& -\frac{\mu}{\kappa_{0}} \bar{V}
\end{aligned}
$$

While the extra shear stress components are

$$
\begin{gathered}
\bar{S}_{\overline{X X}}=2\left(\mu+\frac{1}{\beta \mathrm{c}_{1}}\right) \bar{U}_{\bar{X}}-\frac{1}{3 \beta \mathrm{c}_{1}^{3}}\left[2 \bar{U}_{\bar{X}}^{2}+\left(\bar{U}_{\bar{Y}}+\bar{V}_{\bar{X}}\right)^{2}+2 \bar{V}_{\bar{Y}}^{2}\right] \bar{U}_{\bar{X}} \\
\bar{S}_{\bar{X} \bar{Y}}=\left(\mu+\frac{1}{\beta \mathrm{c}_{1}}\right)\left(\bar{V}_{\bar{X}}+\bar{U}_{\bar{Y}}\right)-\frac{1}{6 \beta c_{1}^{3}} \\
\quad\left[2 \bar{U}_{\bar{X}}^{2}+\left(\bar{U}_{\bar{Y}}+\bar{V}_{\bar{X}}\right)^{2}+2 \bar{V}_{\bar{Y}}^{2}\right]\left(\bar{V}_{\bar{X}}+\bar{U}_{\bar{Y}}\right) \\
\bar{S}_{\overline{Y Y}}=2\left(\mu+\frac{1}{\beta \mathrm{c}_{1}}\right) \bar{V}_{\bar{Y}}-\frac{1}{3 \beta \mathrm{c}_{1}^{3}}\left[2 \bar{U}_{\bar{X}}^{2}+\left(\bar{U}_{\bar{Y}}+\bar{V}_{\bar{X}}\right)^{2}+2 \bar{V}_{\bar{Y}}{ }^{2}\right] \bar{V}_{\bar{Y}}
\end{gathered}
$$

Where $\rho, \bar{U}, \bar{V}, \bar{Y}, \bar{P}, \sigma, \kappa_{0}, \beta_{0}, \beta, c_{1}, \mu$, are the fluid density, axial velocity, transverse velocity, transverse coordinate, the pressure, electrical conductivity, permeability parameter, constant magnetic field, the material constant of Powell-Eyring fluid, dynamic viscosity respectively, and $\bar{S}_{\overline{X X}}, \bar{S}_{\bar{X} \bar{Y}}, \bar{S}_{\bar{Y} \bar{Y}}$ are the components of extra stress tensors.

The subscripts denote the partial derivatives. The flow is time dependent in the laboratory coordinate system $(\bar{X}, \bar{Y}, \bar{t})$ (i.e. the flow is unsteady). However in a coordinate system $(\bar{x}, \bar{y})$ moving with the wave speed $c$ in the positive $\bar{x}$ - direction the motion is stationary. Defining the following transformation

$\bar{x}=\bar{X}-c \bar{t}, \bar{y}=\bar{Y}, \bar{u}=\bar{U}-c, \bar{v}=\bar{V}, \bar{p}(\bar{x}, \bar{y})=\bar{P}(\bar{X}, \bar{Y}, \bar{t})$

Here $\bar{u}, \bar{v}, \bar{P}$, designate the velocity component and the pressure in wave frame.

Hence the governing equations in wave frame is scripted as

$$
\begin{gathered}
\frac{\partial \bar{u}}{\partial \bar{x}}+\frac{\partial \bar{v}}{\partial \bar{y}}=0 \\
\rho\left((\bar{u}+c) \frac{\partial \bar{u}}{\partial \bar{x}}+\bar{v} \frac{\partial \bar{u}}{\partial \bar{y}}\right)=-\frac{\partial \bar{p}}{\partial \bar{x}}+\frac{\partial \bar{S}_{\overline{x x}}}{\partial \bar{x}}+\frac{\partial \bar{S}_{\overline{x y}}}{\partial \bar{y}} \\
-\sigma \beta_{0}^{2}(\bar{u}+c)-\frac{\mu}{\kappa_{0}}(\bar{u}+c) \\
\rho\left((\bar{u}+c) \frac{\partial \bar{v}}{\partial \bar{x}}+\bar{v} \frac{\partial \bar{v}}{\partial \bar{y}}\right)=-\frac{\partial \bar{p}}{\partial \bar{y}}+\frac{\partial \bar{S}_{\overline{y x}}}{\partial \bar{x}}+\frac{\partial \bar{S}_{\overline{y y}}}{\partial \bar{y}}-\frac{\mu}{\kappa_{0}} \bar{v}
\end{gathered}
$$

To figure out the non- dimensional analysis we consider the non- dimensional quantities as follows:

The $\bar{Y}$-component of momentum equation 


\section{International Journal of Science and Research (IJSR) \\ ISSN (Online): 2319-7064}

Index Copernicus Value (2016): 79.57 | Impact Factor (2015): 6.391

$x=\frac{2 \pi \bar{x}}{\lambda}, y=\frac{\bar{y}}{d}, u=\frac{\bar{u}}{c}, v=\frac{\bar{v}}{c}, h=\frac{\bar{H}}{d}, \kappa=\frac{\kappa_{0}}{d}$

$p=\frac{2 \pi d^{2} \bar{P}}{\lambda \mu c}, \delta=\frac{2 \pi d}{\lambda}, S=\frac{d \bar{S}}{\mu c}, \phi=\frac{b}{d}, \operatorname{Re}=\frac{2 \pi \rho c d}{\mu}$,

$W=\frac{1}{\mu \beta c_{1}}, A=\frac{W}{6}\left(\frac{c}{c_{1} d}\right)^{2}, M=\frac{\sigma \beta^{2} d^{2}}{\mu}, m^{2}=\left(M+\frac{1}{\kappa}\right)$

$\delta, \operatorname{Re}, M, \phi, W, \kappa, A$, are the wave number, Renold number, the Hartman number, amplitude ratio, the dimensionless permeability of the porous medium parameter and the two last parameters denote the material fluid parameters.

By introducing the relationship between the stream function $\psi(x, y)$ and the velocity components as

$$
u=\frac{\partial \psi}{\partial y} \text { and } \quad v=-\delta \frac{\partial \psi}{\partial x}
$$

By employing the above quantities listed in Eqs .(17), (18) the continuity equation (14) is automatically satisfied while the non- dimensional form of the momentum equations, Eqs.(15),(16) in terms of stream function under the assumption of long wavelength and low Renold are

$$
\begin{aligned}
& -P_{x}+\frac{\partial S_{x y}}{\partial y}-m^{2}\left(\psi_{y}+1\right)=0 \\
& P_{y}=0 \\
& S_{x y}=(1+W) \psi_{y y}-A\left(\psi_{y y}\right)^{3}
\end{aligned}
$$

The non- dimensional boundary conditions with respect to long wavelength and low Renold number are

$$
\begin{aligned}
& \psi=0, \psi_{y y}=0 \quad \text { at } y=0 \\
& \psi_{y}=-1-\alpha S_{x y}, \psi=F \quad \text { at } y=h(x) \\
& \text { and } \\
& h(x)= \pm(1+\phi \sin x)
\end{aligned}
$$

Where $\alpha$ is the slip parameter, $F$ is the dimensionless time mean flow rate in the wave frame, $h(x)$ non- dimensional wall equation.

\section{Rate of Volume Flow}

The instantaneous volume flow rate in the fixed frame of coordinate $(\bar{X}, \bar{Y}, \bar{t})$ is given by

$$
\bar{Q}=\int_{0}^{\bar{H}(\bar{X}, \bar{Y}, \bar{t})} \bar{U}(\bar{X}, \bar{Y}, \bar{t}) d \bar{Y}
$$

While in the wave frame the flow becomes steady with respect to the reference $(\bar{x}, \bar{y})$. The expression of the volumetric flow rate becomes

$$
\bar{q}=\int_{0}^{\bar{h}(\bar{x}, \bar{y}, \bar{t})} \bar{u}(\bar{x}, \bar{y}, \bar{t}) d \bar{y}
$$

Using the transformations in Eq.(13) into Eq.(25) and with aid of Eq.(26) the relation between the volumetric flow rate in two frames is

$$
\bar{Q}=\int_{0}^{\bar{h}}(\bar{u}+c) d \bar{y}=\int_{0}^{\bar{h}} \bar{u}(\bar{x}, \bar{y}) d \bar{y}+\int_{0}^{\bar{h}} c d \bar{y}=\bar{q}+c \bar{h}
$$

The time- mean flow over one period $\left(T=\frac{\lambda}{c}\right)$ of the peristaltic wave is defined as

$$
Q^{*}=\int_{0}^{T} \bar{Q} d \bar{t}=\frac{1}{T} \int_{0}^{T}(\bar{q}+c \bar{h}) d \bar{t}
$$

By using Eq.(27), Eq. (28) will be

$$
Q^{*}=\bar{q}+c d
$$

Introducing the non- dimensional time- mean flow $\theta$ and $F$ respectively in the fixed and wave frames of coordinate as

$\theta=\frac{Q^{*}}{c d} \quad$ and $\quad F=\frac{\bar{q}}{c d}$

where

$F=\int_{0}^{h} \frac{\partial \psi}{\partial y} d y=\psi(h)-\psi(0)$

The non- dimensional form of Eq.(27) is

$$
Q=\int_{0}^{h} u d y
$$

Employing Eq.(30) we derive the following dimensionless relationship

$\theta=F+1$

The expression of non- dimensional pressure difference $\Delta P_{\lambda}$ per wavelength is

$\Delta p_{\lambda}=\int_{0}^{2 \pi} \frac{d p}{d x} d x$

\section{Solution Technique}

Employing the perturbation technique for obtaining the solution of the system for a small Powell- Eyring fluid parameter $A$

$$
\begin{aligned}
& \psi=\psi_{0}+A \psi_{1}+O\left(A^{2}\right) \\
& F=F_{0}+A F_{1}+O\left(A^{2}\right)
\end{aligned}
$$

On differentiating Eq.(19) w.r.t $y$ and take into consideration Eq.(20), a non- linear system of partial differential equation deduced as

$$
\frac{\partial^{2} S_{x y}}{\partial y^{2}}-m^{2} \psi_{y y}=0
$$

Now substituting Eq.(35) into Eq.(37), one can obtain the following system of partial differential equations associated with boundary conditions as below 


\section{International Journal of Science and Research (IJSR) \\ ISSN (Online): 2319-7064}

Index Copernicus Value (2016): 79.57 | Impact Factor (2015): 6.391

\subsection{Zeroth Order System}

The corresponding system at zero order is

$$
(1+W) \frac{\partial^{4} \psi_{0}}{\partial y^{4}}-m^{2} \psi_{0 y y}=0
$$

Associated with the boundary conditions

$\psi_{0}=0, \psi_{0 y y}=0 \quad$ at $y=0$

$\psi_{0}=F_{0}, \psi_{0 y}=-1-\alpha(1+W) \psi_{0 y y} \quad$ at $y=h(x)$

Solving Eq.(38), the solution is taken the below form

$\psi_{0}=\frac{1}{N_{1}^{2}}\left(e^{N_{1} y} c_{1}+e^{-N_{1} y} c_{2}\right)+c_{3}+c_{4} y$

\subsection{First Order System}

The concluded first order system is

$(1+W) \frac{\partial^{4} \psi_{1}}{\partial y^{4}}-m^{2} \psi_{1 y y}+\frac{\partial^{2}}{\partial y^{2}}\left(\psi_{0 y y}\right)^{3}=0$

Companied with the boundary conditions

$\psi_{1}=0, \psi_{1 y y}=0 \quad$ at $y=0$

$\psi_{1}=F_{1}, \psi_{1 y}=-\alpha(1+W) \psi_{1 y y}+\alpha\left(\psi_{0 y y}\right)^{3}$

at $y=h(x)$

In solving the above system the first order solution is determined as

$\psi_{1}=\frac{1}{N_{1}^{2}} e^{-3 N_{1} y}\left(L_{1}+L_{2}+8 m^{2} e^{\frac{2 m y}{\sqrt{1+W}}}\left(e^{\frac{2 m y}{\sqrt{1+W}}} c_{5}+c_{6}\right)\right)+c_{7}+c_{8} y$

where

$$
\begin{aligned}
& N_{1}=\frac{m}{\sqrt{(1+W)}}, \\
& L_{1}=N_{1}^{2}\left(c_{2}^{3}+6 c_{2} c_{1}^{2} e^{4 N_{1} y}\left(-5+2 N_{1} y\right)+c_{1}^{3} e^{6 N_{1} y}\right) \\
& L_{2}=-6 N_{1}^{2} c_{1} c_{2}^{2} e^{2 N_{1} y}\left(5+2 N_{1} y\right)
\end{aligned}
$$

In which $\left(c_{1}, c_{2}, c_{3}, c_{4}, c_{5}, c_{6}, c_{7}, c_{8}\right)$ can be calculated by applying Mathematica 9 programs.

\section{Results and Discussion}

In this section the graphical and numerical results of all the pertinent parameters are discussed and plotted for velocity profile, pressure rise, pressure gradient, the local shear stress on the wall $y=h$ and the trapping phenomenon of the peristaltic flow through the following subsections.

\subsection{Velocity Profile}

The outcomes of axial velocity $u(y)$ for a fixed value of $x=0.2$ in terms of emerging parameters have been plotted in this part, and it is clear from the graphs that the velocity profile attains parabolic in nature, also another observation can be made which is the maximum value of the velocity attains in the central part of the channel whereas the situation is reversed toward the walls of the channel. Figure.1(a). Describes the behavior of axial velocity with variation of velocity slip parameter $(\alpha)$, it shown that any intensification in $(\alpha)$ amount produce decreasing in the velocity profile. Figure 1(b), and Figure 2(a), study the effect of EyringPowell fluid parameters $A$ and $W$ on velocity axial. The graphs show the two parameters have the same effect on the velocity (i.e. increasing their amount reduces the velocity in return). Figures 2(b) and Figure 3(a), transmit reduction in the velocity as the magnitude of magnetic field $(M)$ and the amplitude ratio $\phi$ parameters enlarge which means the magnetic field acts as a retarded force. Increasing behavior of velocity axial is noticed as the porosity parameter $\kappa$ enhances due to Figure 3(b).

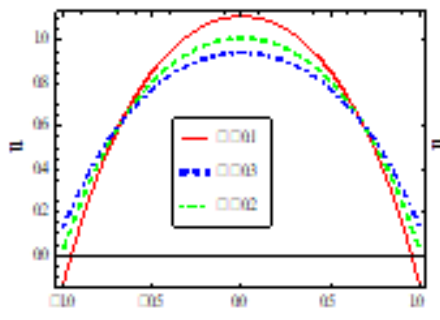

(a)

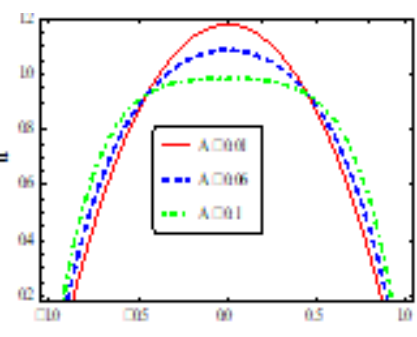

(b)
Figure 1: Velocity profile via variation of (a) Slip parameter (b) Fluid parameter, and fixed data $(M=0.4, \kappa=0.7, \phi=0.6, \theta=1.7, W=0.2)$

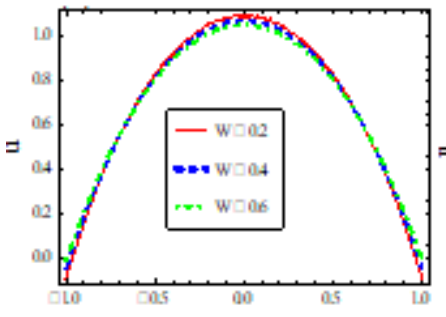

(a)

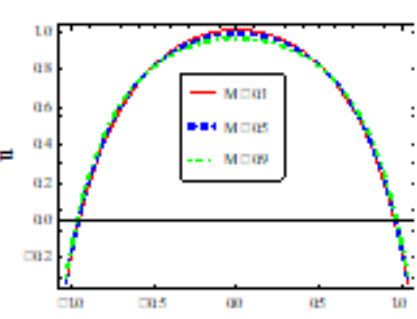

(b)
Figure 2: Velocity profile via variation of (a) Fluid parameter (b) Magnetic field parameter, and fixed data ( $\alpha=0.1, \kappa=0.7, \phi=0.6, \theta=1.7, A=0.01)$

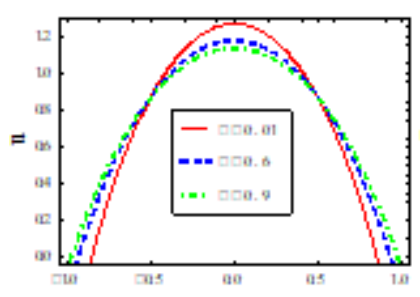

(a)

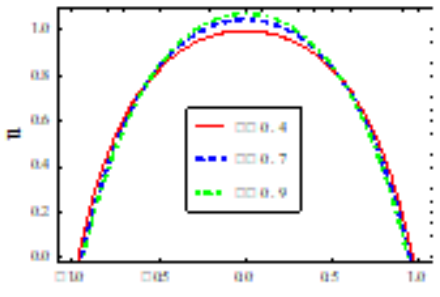

(b)
Figure 3: Velocity profile via variation of (a) Amplitude ratio parameter (b) Porosity parameter, and fixed data ( $\alpha=0.1, W=0.7, M=0.4, \theta=1.7, A=0.01)$

\subsection{Pressure gradient}

In this part of the discussion we recorded the reaction of pressure gradient with the variation of different interesting parameters. Figure 4(a), demonstrate that when the amount of $(\alpha)$ coefficient arises the pressure gradient value in return get smaller in the central part of the channel this means a

\section{Volume 6 Issue 12, December 2017}

\section{www.ijsr.net}




\section{International Journal of Science and Research (IJSR) \\ ISSN (Online): 2319-7064}

Index Copernicus Value (2016): 79.57 | Impact Factor (2015): 6.391

great flow can pass with no need to impose large pressure in the central part of the channel. Whereas larger pressure gradient imposed near the walls of the channel to attains the same flow to pass through it. Figure 4(b), and Figure 5(a), reflect totally opposite influences of fluid parameters $(W)$ and $(A)$ upon the pressure gradient profile. (i.e. when $(W)$ increases the amount of $\frac{d P}{d x}$ increases to reach a fixed values at $-1 \leq y \leq 1$ in the central part of the channel while increasing in $(A)$ values decreases the pressure gradient. Figure 5(b), portrays that for ascending value of magnetic parameter $(M)$ provides resistance to flow rate and hence pressure gradient increases to a certain value in the center while the result reversed near the walls. Figure 6(a), and Figure 6(b), illustrate the impact of amplitude ratio $(\phi)$ and porosity parameter $\kappa$ on pressure gradient. It can be observed larger values of $(\phi)$ and $(\kappa)$ values cause decreases in $\frac{d P}{d x}$ value in the central part of the channel while reduction function of pressure is seen near the walls.

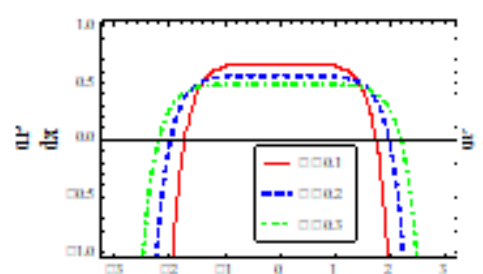

(a)

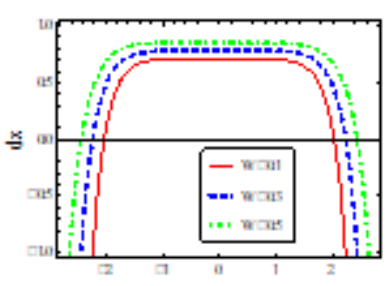

(b)
Figure 4: Pressure gradient for variation of (a) Slip parameter (b)Fluid parameter, and for fixed data $(M=0.2, \kappa=1.5, \phi=0.2, \theta=-0.26, A=0.2)$

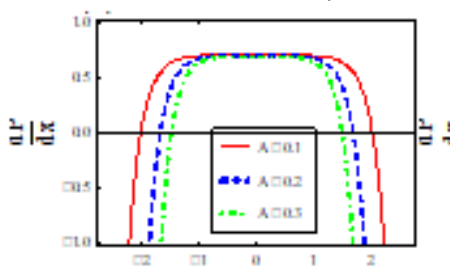

(a)

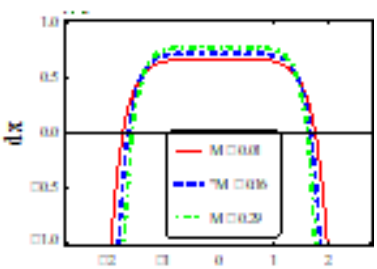

(b)
Figure 5: Pressure gradient for variation of (a) Fluid parameter (b) Magnetic field parameter, and for fixed data $(\alpha=0.1, \kappa=1.5, \phi=0.2, \theta=-0.26, W=0.1)$

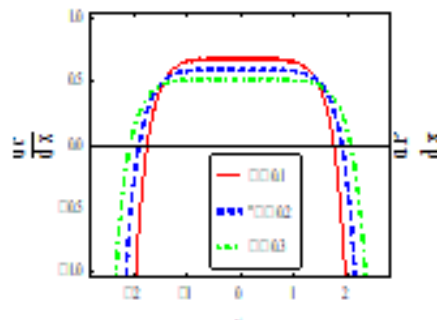

(a)

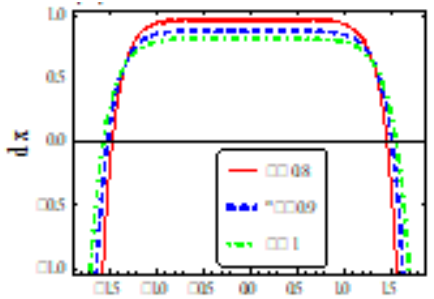

(b)
Figure 6: Velocity profile via variation of (a) Amplitude ratio parameter (b) Porosity parameter, and fixed data $(\alpha=0.1, W=0.1, M=0.2, \theta=-0.26, A=0.2$ )

\subsection{Shear Stress}

The purpose of this subsection is to investigate the influence of various parameters on local shear stress on the wall $y=h$. Figure 7(a), exhibits the effect of slip coefficient $(\alpha)$ on absolute value of shear stress $\left(S_{x y}\right)$. It is shown that when $(\alpha)$ increases for $0 \leq y \leq 1.9$ the value of $\left(S_{x y}\right)$ decreases while for $2 \leq y \leq 3$ the value of $\left(S_{x y}\right)$ increases. Figure 7(b), and Figure 8(a), indicate an opposite impact for fluid parameters $(A)$ and $(W)$ on magnitude of $\left(S_{x y}\right)$. Ascending value of $(A)$ parameter reduces $\left(S_{x y}\right)$ magnitude, while for larger magnitude of $(W)$ enhances the shear stress on the wall. Figure 8(b), discloses the impact of amplitude ratio $(\phi)$ quantity on local shear stress. It is noticed that an increase in $(\phi)$ causes decreasing in the shear stress on the wall in the region $0 \leq y \leq 2$ while it is increasing after this values till the wall $y=h$. Mixed behavior for axial shear stress deduced from Figure 9 (a), and (b) upon increasing of magnetic field parameter $(M)$ and porosity parameter $(\kappa)$.

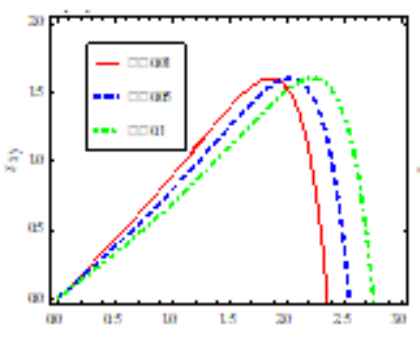

(a)

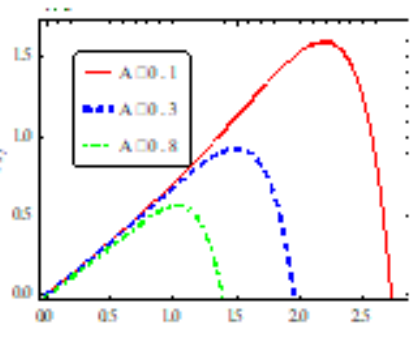

(b)
Figure 7: Shear Stress via Variation of (a) Slip parameter (b)Fluid parameter, and for fixed data $(M=0.02, \kappa=1.5, \phi=0.2, \theta=-0.26, A=0.1)$

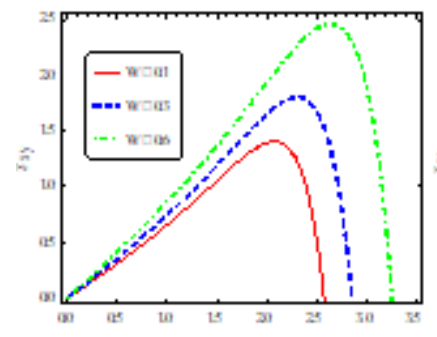

(a)

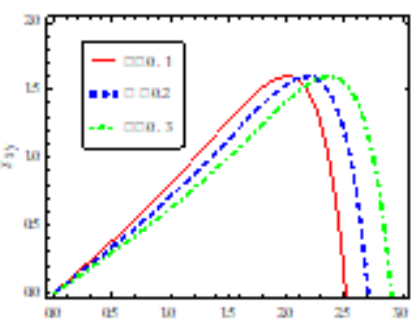

(b)
Figure 8: Shear Stress via Variation of (a) Amplitude ratio parameter (b)Fluid parameter, and for fixed data $(M=0.02, \kappa=1.5, \alpha=0.2, \theta=-0.26, W=0.1)$

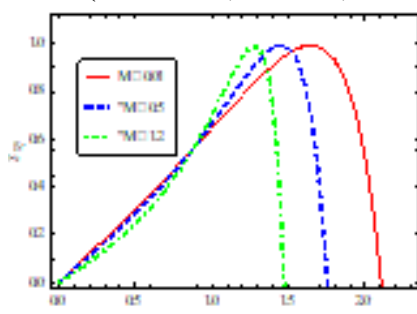

(a)

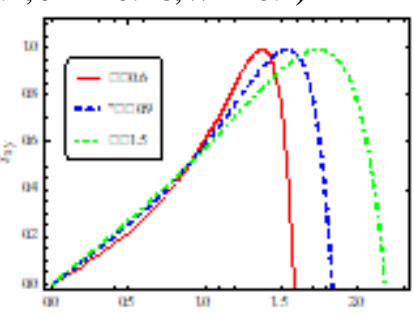

(b)
Figure 9: Shear Stress via Variation of (a) Magnetic field parameter (b) Porosity parameter, and for fixed data 


\section{International Journal of Science and Research (IJSR) \\ ISSN (Online): 2319-7064}

Index Copernicus Value (2016): 79.57 | Impact Factor (2015): 6.391

$(A=0.2, \phi=0.2, \alpha=0.2, \theta=-0.26, W=0.1)$

\subsection{Trapping Phenomenon}

Figure 10, deduced that for larger values of slip parameter $(\alpha)$ the size and the number of trapped bolus is seen to increase in the central part of the channel. Figure 11, displays the impact of fluid parameter $(A)$ on the streamlines. The size and the number of the bolus decreases with an increase value of $(A)$. Moreover an opposite effect is observed on the streamlines with ascending value of fluid parameter $(W)$ see Figure 12. However Figure 13 is sketched to study the impact of magnetic field parameter $(M)$ on the trapping. It is clear from the plots that larger magnitudes of $(M)$ reduces the flow and hence the size of the bolus get smaller and then disappear. Figure 14, portrays the enhancing reaction on streamlines as the amplitude ratio $(\phi)$ increases (i.e. the size of the bolus becomes bigger with ascending value of $(\phi)$ ) Moreover the size and the number of trapped bolus increase as the porosity parameter $(\kappa)$ increase via Figure 15.

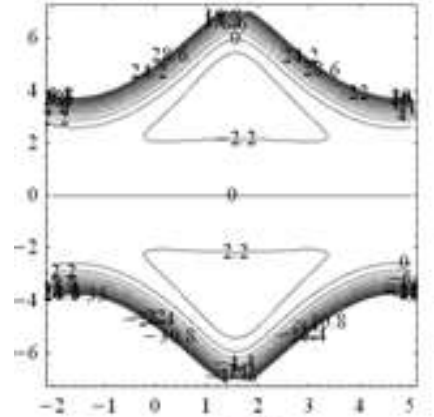

(a)

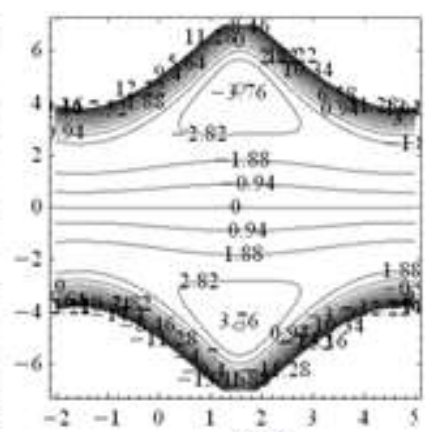

(b)
Figure 10: Streamlines for variation of slip parameter (a) $\alpha=0.3$ (b) $\alpha=0.9$ and for fixed parameters $(M=0.01, \phi=0.2, A=0.1, \theta=-0.26, W=0.8, \kappa=0.9)$

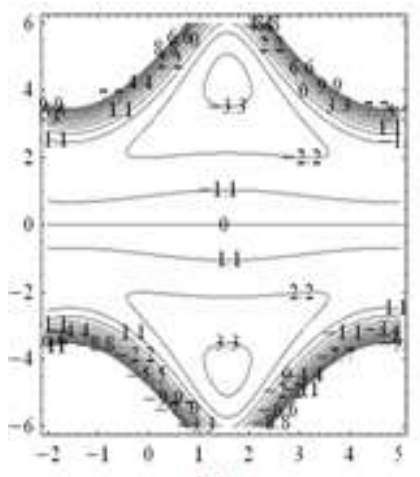

(a)

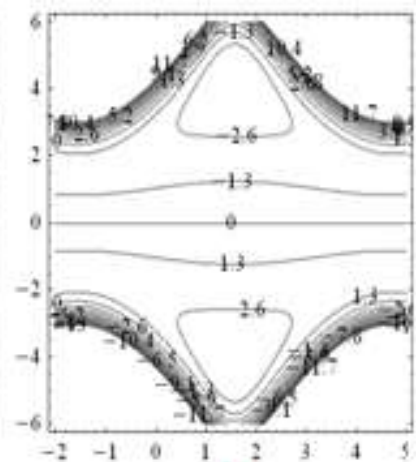

(b)
Figure 11: Streamlines for variation of Fluid parameter $\begin{array}{ll}\text { (a) } A=0.1 & \text { (b) } A=0.6 \text {, and for fixed parameters }\end{array}$ $(M=0.01, \phi=0.2, \alpha=0.3, \theta=-0.26, W=0.8, \kappa=0.9)$

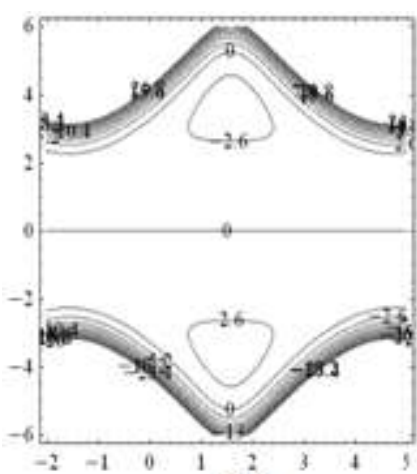

(a)

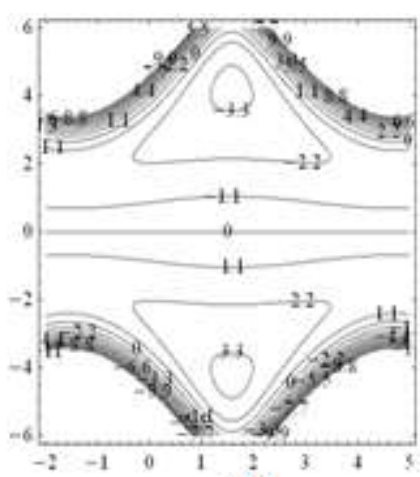

(b)
Figure 12: Streamlines for variation of fluid parameter (a) $W=0.19$ (b) $W=0.7$, and for fixed parameters

$(M=0.01, \phi=0.2, A=0.1, \theta=-0.26, \alpha=0.1, \kappa=0.9)$

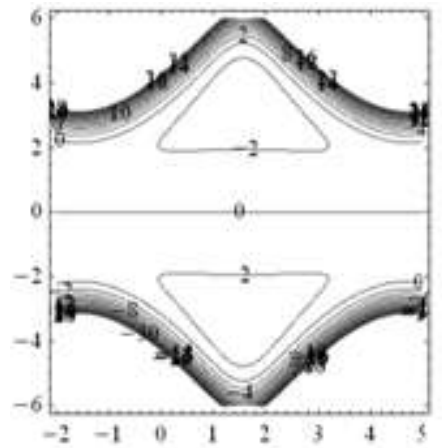

(a)

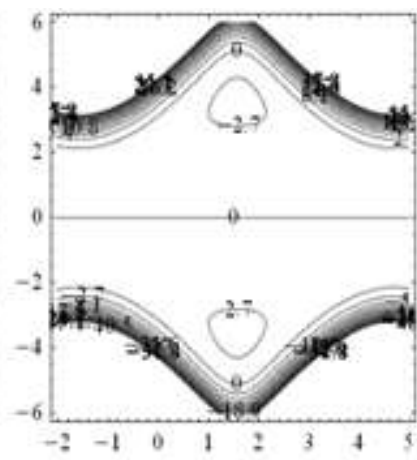

(b)
Figure 13: Streamlines for variation of magnetic field parameter (a) $M=0.01$ (b) $M=0.06$, and for fixed parameters $\quad(W=0.8, \phi=0.2, A=0.1, \theta=-0.26, \alpha=0.2, \kappa=0.9)$

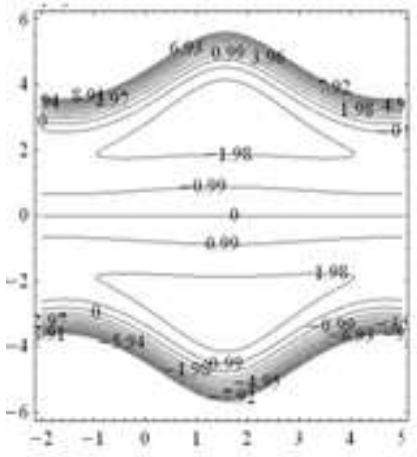

(a)

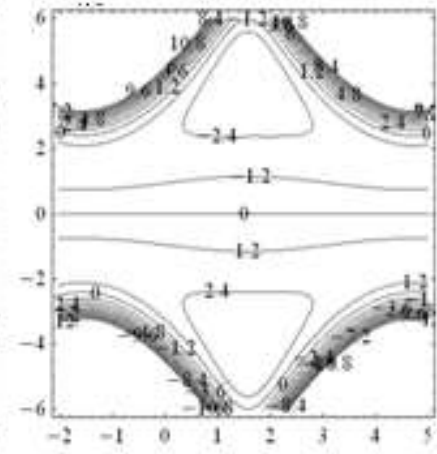

(b)
Figure 14: Streamlines for variation of amplitude ratio parameter (a) $\phi=0.13$ (b) $\phi=0.21$, and for fixed parameters $\quad(W=0.8, M=0.01, A=0.1, \theta=-0.26, \alpha=0.2, \kappa=0.9)$

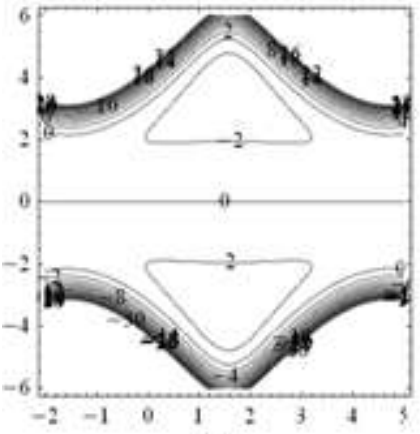

(a)

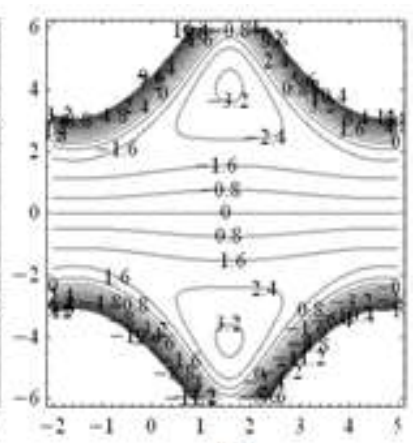

(b)
Figure 15: Streamlines for variation of porosity parameter

\section{Volume 6 Issue 12, December 2017}

\section{www.ijsr.net}




\section{International Journal of Science and Research (IJSR) \\ ISSN (Online): 2319-7064}

Index Copernicus Value (2016): 79.57 | Impact Factor (2015): 6.391

(a) $\kappa=0.13$ (b) $\kappa=0.21$, and for fixed

parameters $\quad(W=0.8, M=0.01, A=0.1, \theta=-0.26, \alpha=0.2, \phi=0.2)$

\subsection{Pressure Rise}

In this subsection we testify the relationship that relates the non- dimensional average pressure rise per wavelength and dimensionless mean flow $\operatorname{rate}(\theta)$ versus variation in interesting parameters included in $\left(\Delta P_{\lambda}\right)$. The final expression for $\left(\Delta P_{\lambda}\right)$ is obtained by a numerical integration of a series approximation for $\frac{d P}{d x}$ using Mathematica program. Plots show a linear relationship between the flow rate and $\left(\Delta P_{\lambda}\right)$ also the pumping region divided into four partitions The positive pumping with $(\Delta P>0, \theta>0)$ which represents the peristaltic pumping. The second region is called co-pumping region or (augmented flow) creates for $(\Delta P<0, \theta<0)$. The third region where there is no flow recognized when $(\Delta P<0, \theta<0)$, and free pumping region achieved when $\left(\Delta P_{\lambda}=0\right)$. Figure 16(a), shows that when the magnetic field parameter $(M)$ increases the pumping rate decreases in all regions. It visualized from Figure 16(b), that when the slip coefficient $(\alpha)$ increases the pumping rate enhances in the co-pumping region however opposite result noticed in peristaltic flow i.e. the pumping decreases. Figure 17(a), illustrates the effect of amplitude ratio $(\phi)$ on pumping. It has seen for larger value of $(\phi)$ the pumping decreases. Figure 17(b), deduces the reaction on the pumping via variation of fluid parameter $(W)$. The copumping region and free pumping decreases for ascending value of $(W)$ whereas enhances the peristaltic flow. For larger values of $(A)$ the peristaltic flow increases however the co- pumping region reveals mixed behavior. Reduction in free pumping is noticed too see Figure 18(a). It is observed from Figure 18(b), that the pressure rise increases in all regions with an increasing the porosity parameter $(\kappa)$.

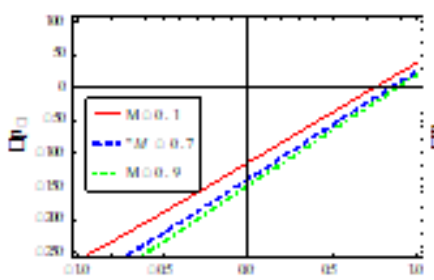

(a)

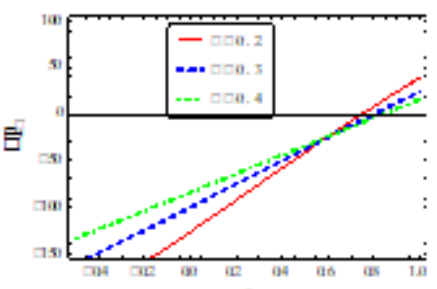

(b)
Figure 16: Pressure rise upon Variation of (a) Magnetic field parameter (b) slip parameter, and fixed parameter $(W=0.8, \kappa=0.9, A=0.1, \theta=-0.26, \phi=0.2)$

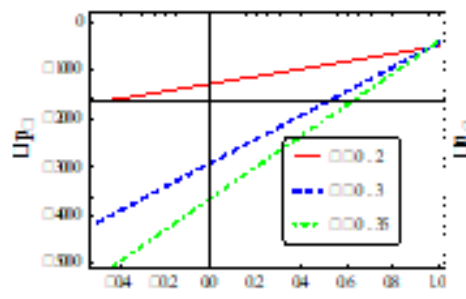

(a)

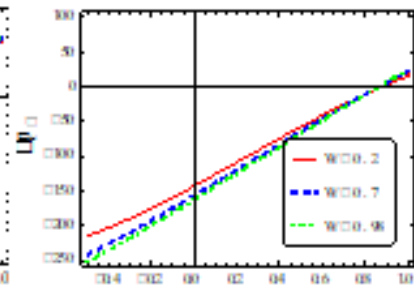

(b)
Figure 17: Pressure rise upon Variation of (a) Amplitude ratio parameter (b) fluid parameter, and fixed parameter $(\alpha=0.2, \kappa=0.9, A=0.1, \theta=-0.26, \phi=0.2, W=0.8$ )

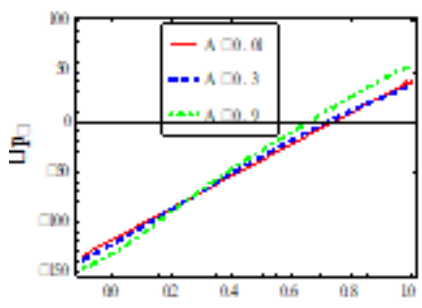

(a)

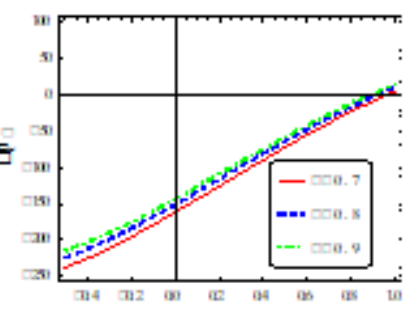

(b)
Figure 18: Pressure rise upon Variation of (a) fluid parameter (b) porosity parameter, and fixed parameter $(\alpha=0.2, W=0.8, A=0.1, \theta=-0.26, \phi=0.2)$

\section{Conclusion}

In this paper, the slip effect on MHD peristaltic flow of Powell- Eyring fluid in a porous medium through a symmetric channel is investigated. The solution is obtained by adopting assumptions of long wavelength approximation and low Renolds number. Main finding remarks can be made as follows:

1) Velocity axial has a diminishing behavior due an increase in slip parameter $(\alpha)$ in the central part of the channel whereas it is different towards walls.

2) The influences of Hartman number $(M)$ and porosity parameter $(\kappa)$ on the solution expressions are qualitatively opposite.

3) A mixed behavior of local shear stress on the wall $y=h$, is noticed due to increase of amplitude ratio magnitude $(\phi)$.

4) Pressure gradient magnitude has increasing function due to fluid parameter $W$ whereas it decreases with parameter $(A)$. Mixed behavior is noticed via parameters $(\alpha),(\phi),(\kappa)$ and $(M)$

5) The size and numbers of trapped bolus increase due to $W$ but decrease via $(A)$.

6) In the pumping region, the pumping rate increases via increasing $(A)$, while the co- pumping and free pumping region increase with enhancement of $(\alpha)$, and $(\kappa)$.

\section{References}

[1] T.W. Latham, Fluid motion in a peristaltic pump, M. S. Thesis, Massachusetts Institute of Technology, Cambridge, 1966.

\section{Volume 6 Issue 12, December 2017}




\section{International Journal of Science and Research (IJSR) \\ ISSN (Online): 2319-7064}

Index Copernicus Value (2016): 79.57 | Impact Factor (2015): 6.391

[2] A.H. Shapiro, M.Y. Jaffrin, and S.L. Wienberg "Peristaltic pumping with long wavelengths at low Reynolds number". Journal of Fluid Mechanic,(37), pp. 799-825, 1969.

[3] M. Devakar, K. Ramesh, S. Chouhan, and A. Raje, “ Fully developed flow of non- Newtonian fluids in a straight uniform square duct through porous medium", Journal of the Association of Arab Universities for Basic and Applied Science, pp. 1815-3852, 2016.

[4] G. Ismail, F. Darwesh, , N. Dabe,“ Peristaltic Transport of a Magneto Non- Newtonian Fluid through A porous medium in horizontal Finite channel", IOSR Journal of Mathematics, (8), pp. 32-39, 2013.

[5] A. Khan, R. Ellahi, ,and M. Usman," The Effects of Variable Viscosity on The Peristaltic Flow of NonNewtonian Fluid Through A Porous Medium in an Inclined Channel with Slip Boundary Conditions", Journal of Porous Media, (16), 2013.

[6] F. Oyelami, , M. Dada, "Transient Magnetohydrodynamic Flow of Eyring- Powell Fluid in A Porous Medium", Ife Journal of Science, 18(2), 2016.

[7] M. Abbas, M. Bhatti, "Simultaneous effect of slip and MHD on peristaltic blood flow of Jeffrey fluid model through a porous medium", Alexandria Engineering. Journal, 55, pp. 1017- 1023, 2016.

[8] A. Tanveer, A. Alsaedi, , M. Shafique, T. Hayat, "Magnetohydrodynamic effects on peristaltic flow of hyperbolic tangent nanofluid with slip conditions and joule heating in an inclined channel", International Journal of Heat and Mass Transfer 102, pp. 54-63, 2016

[9] M. Veerakrishna, B. Swarnalathamma, " Convective Heat and Mass Transfer on MHD Peristaltic flow of Williamson Fluid with the Effect of Inclined Magnetic Field", International Conference on. Condensed Matter and Applied Physics, pp. 020461-1-020461-8, 2015.

[10]F. Abbasi, T. Hayat, B. Ahmad, "Hydromagnetic Peristaltic Transport of Variable Viscosity Fluid with Heat Transfer and Porous Medium", Applied Mathematics and. Information Sciences, 10, pp. 2173 2181, 2016.

[11] T. Hayat, Q. Hussain, N. Ali,“ Influence of partial slip on the peristaltic flow in a porous medium", Physica A, 387, pp. 3399-3409, 2008.

[12] S. Hina, “ MHD peristaltic transport of Eyring-Powell fluid with heat/mass transfer, wall properties and slip conditions", Journal of Magnetism and Magnetic Materials, 404, pp. 148-158, 2016.

[13] T. Hayat, S. Shah, B. Ahmad, M. Mustafa, “ Effect of Slip on peristaltic flow of Powell- Eyring fluid in a symmetric channel", Applied Bionics and Biomechanics, 11, pp. 69- 79, 2014.

\section{Author Profile}

Ahmad M. Abdulhadi I received the B.S and MS.c degree from Baghdad university -college of sciencedept.of mathematics 1988. The Ph.D from pune univeristy-India in 2000.I have degree of professor in mathematics since 2012.Now,working in college of science-department. Of mathematics.

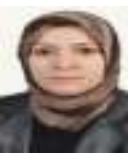

Hayat Ali: I received the B.S. and M.S degrees in Applied Mathematics from University of Technology in 2001 and 2007, respectively. In 2007 I appointed as a lecturer in Department of Applied Science University of Technology. She is now Associative Professor in the same Department.

Volume 6 Issue 12, December 2017

www.ijsr.net

Licensed Under Creative Commons Attribution CC BY 